\title{
Dynamical theory for diffractive x-ray imaging of one-dimensional periodic objects
}

\author{
K. Nygård, ${ }^{1, a)}$ D. K. Satapathy, ${ }^{1}$ O. Bunk, ${ }^{1}$ F. Pfeiffer, ${ }^{1,2}$ C. David, ${ }^{1}$ and J. F. van der Veen ${ }^{1,3}$ \\ ${ }^{1}$ Paul Scherrer Institut, CH-5232 Villigen PSI, Switzerland \\ ${ }^{2}$ École Polytechnique Fédérale de Lausanne, CH-1015 Lausanne, Switzerland \\ ${ }^{3}$ ETH Zürich, $\mathrm{CH}-8093$ Zürich, Switzerland
}

(Received 2 April 2008; accepted 10 May 2008; published online 28 May 2008)

\begin{abstract}
A dynamical theory for diffractive $\mathrm{x}$-ray imaging of one-dimensional periodic objects is derived by solving the Helmholtz equation in the parabolic approximation using the coupled-wave theory. A method to account for volume-diffraction effects, based on propagating backwards the eigenmodes of the microfluidic array, is demonstrated for a colloidal suspension in confinement.
\end{abstract}

(C) 2008 American Institute of Physics. [DOI: 10.1063/1.2938050]

Diffractive $\mathrm{x}$-ray imaging is emerging as a promising tool for nanometer-scale microscopy of both hard and soft condensed matter. Subsequent to the proof-of-principle experiments on manufactured specimens, ${ }^{1}$ the field has rapidly evolved with the application to, e.g., deformation fields inside nanocrystals, ${ }^{2}$ single cells, ${ }^{3}$ and, as a special case utilizing only partially coherent illumination, confinementinduced ordering of colloidal suspensions. ${ }^{4}$

The application of diffractive $\mathrm{x}$-ray imaging to extended objects raises, however, an issue hitherto largely neglected: the so-called volume-diffraction effects (i.e., the wave field inside the object differing from the incident plane wave). Since the technique relies on determining the object from the reconstructed complex wave field, these effects will introduce artifacts in the image. For yeast cells, the volumediffraction effects have been studied using multislice simulations and, in a perturbative manner, within the Rytov approximation, ${ }^{5}$ whereas refraction inside nanocrystals has been accounted for within the distorted-wave Born approximation. ${ }^{6}$ In the case of confined colloids, the aforementioned effects have been minimized using an ad hoc approximation of propagating the exit wave field backwards through free space. ${ }^{7}$ Nonetheless, a formally exact method to account for volume-diffraction effects is yet to be presented.

In this Letter, we derive a dynamical theory for diffractive $x$-ray imaging of one-dimensional periodic objects. The method is based on casting the Helmholtz equation in the parabolic approximation and solving it in terms of the eigenmodes of the object. This approach has previously been utilized for studies on the beam propagation in and focusing properties of Fresnel zone plates ${ }^{8-10}$ and x-ray waveguides. ${ }^{11}$ Finally, a method to account for volume-diffraction effects, based on backward propagation of the exit wave field through the sample using the eigenmodes of the container, is demonstrated for a colloidal suspension confined in a microfluidic array.

The propagation of monochromatic $\mathrm{x}$ rays through a linear phase grating can be described by the scalar Helmholtz equation in the parabolic approximation,

$$
-\frac{1}{2 k^{2}} \frac{\partial^{2} \Psi}{\partial x^{2}}+\delta \Psi=-\frac{i}{k} \frac{\partial \Psi}{\partial z} .
$$

Here $\delta=1-n$ denotes the spatially varying decrement of the refractive index (neglecting absorption), $k=2 \pi / \lambda$ the magnitude of the wave vector (throughout this study we assume the wavelength $\lambda=1.05 \AA$ ), and $\Psi$ a slowly varying scalar wave field, which represents the component $E_{y}$ of the electric field $\mathbf{E}=\left(0, E_{y}, 0\right)$ parallel to the gratings. We note that the parabolic approximation is accurate for (i) small variations in the refractive index $(\Delta n / n \ll 1)$ and (ii) a narrow angular field $\left(k_{x} / k \ll 1\right)$, both of which are valid for the problem considered. The reader is referred to Fig. 1 for the geometric definitions and the nomenclature used. In what follows, we assume the incident $\mathrm{x}$ rays to travel parallel to the $z$ axis.

Initially, by assuming that the periodic and symmetric refractive index is independent of the longitudinal variable $z$, i.e., $\delta=\delta(x)$ (or equivalently $\theta=0^{\circ}$ ), Eq. (1) can be solved exactly in a straightforward manner. In line with the coupledwave theory outlined in Ref. 8, we expand $\delta(x)$ as a Fourier series,

$$
\begin{aligned}
\delta(x)= & \delta^{\prime}+\Delta \delta\left[1-\frac{w}{p}\right. \\
& \left.-\frac{2 w}{p} \sum_{n=1}^{\infty} \operatorname{sinc}\left(\frac{n \pi w}{p}\right) \cos \left(\frac{n 2 \pi x}{p}\right)\right],
\end{aligned}
$$

with $\delta^{\prime}$ denoting the dispersive (real) part of the refractive

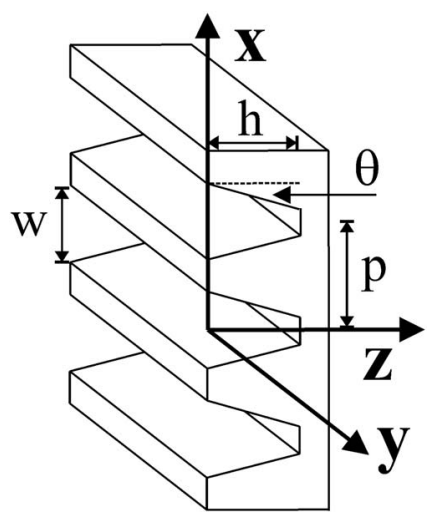

FIG. 1. Definition of coordinate system and variables.

\footnotetext{
${ }^{a)}$ Electronic mail: kim.nygard@psi.ch.
} 


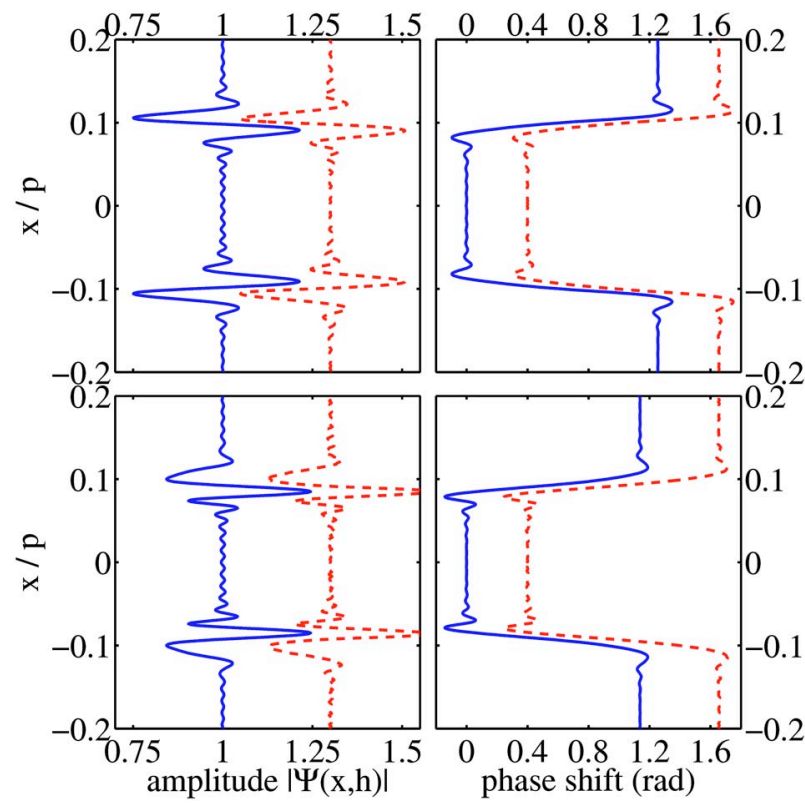

FIG. 2. (Color online) Amplitude (left) and phase-shift (right) profiles of the wave field $\Psi(x, h)$ at the exit of typical Si phase gratings ( $p=1 \mu \mathrm{m}, w$ $=0.2 \mu \mathrm{m}$, and $h=6 \mu \mathrm{m}$ with $\lambda=1.05 \AA$ ). The tapering angles are $\theta=0^{\circ}$ (top) and $\theta=0.1^{\circ}$ (bottom). The solid and dashed lines are obtained using the coupled-wave theory and the finite-difference method, respectively. The profiles are horizontally offset for clarity.

index in the cavity and $\Delta \delta$ the change across the interface. Furthermore, we decompose the wave field in terms of plane waves as

$$
\Psi(x, z)=\sum_{m=-\infty}^{\infty} a_{m}(z) e^{-i m 2 \pi x / p} .
$$

Here the volume-diffraction effects are implicitly included in the longitudinally varying amplitudes $a_{m}(z)$. Hence, we obtain a set of mode-coupling equations as follows:

$$
\begin{aligned}
-\frac{i}{k} \frac{d a_{m}}{d z}= & {\left[\frac{1}{2}\left(\frac{m \lambda}{p}\right)^{2}+\delta^{\prime}+\left(1-\frac{w}{p}\right) \Delta \delta\right] a_{m} } \\
& -\frac{w \Delta \delta}{p} \sum_{n \neq 0} \operatorname{sinc}\left(\frac{n \pi w}{p}\right) a_{m+n} .
\end{aligned}
$$

Although neglected in the present study, ${ }^{12}$ absorption is readily taken into account within this approach by including the imaginary part of the refractive index (i.e., $\delta \rightarrow \delta+i \beta$ using standard notation). The amplitudes $a_{m}(z)[\mathbf{a}(z)$ in vector notation], and hence the wave field $\Psi(x, z)$ according to Eq. (3), are finally obtained by propagating the eigenmodes through the grating, i.e., $\mathbf{a}(z)=\Sigma_{p} \mathbf{c}_{p} \exp \left(i k \lambda_{p} z\right)\left[\mathbf{c}_{p}^{T} \cdot \mathbf{a}(0)\right]$ with $\left\{\mathbf{c}_{p}\right\}$ and $\left\{\lambda_{p}\right\}$ denoting the eigenstates and eigenvalues determined from Eq. (4). ${ }^{13}$ For plane-wave illumination, as in the present case, the boundary conditions are given using the Kronecker delta by $a_{m}(0)=\delta_{m 0}$. Importantly, the integration of Eq. (4) can also be performed in the negative $z$ direction in order to propagate backwards any exit wave field through the grating [using the boundary conditions $a_{m}(h)$ ], a property which will be utilized in the subsequent application to phaseretrieval-based diffractive $x$-ray imaging.

The amplitude and phase-shift profiles of the wave field at the exit of typical Si phase gratings, as given by the above coupled-wave theory, are shown in Fig. 2. Notably, Fresnel fringes, which in the Fraunhofer far-field regime will develop into diffraction maxima, are manifest in the vicinity of the interfaces. Following Ref. 14, these are attributed to the superposition of diffracted edge waves and so-called head waves, with the latter being refracted from the interface at the critical angle of total reflection $\theta_{c} \approx \sqrt{2 \Delta \delta}$. However, the phase gratings considered in the present work are too shallow to exhibit waveguiding effects. We note that these volume-diffraction effects hamper accurate extraction of the electron density from $\mathrm{x}$-ray diffraction data by means of phase-retrieval algorithms.

The actual gratings typically have a tapering angle; in the case of microfluidic arrays, the value $\theta \approx \pm 0.1^{\circ}$ has been reported $^{15}$ (the negative sign corresponding to a rotation by $180^{\circ}$ about the $x$ axis). Strictly speaking, no eigenmodes exist in this case. Nevertheless, the wave field can be solved in the local normal mode basis. ${ }^{16}$ Within this approach, the wave field at each $z$ is expanded in terms of instantaneous eigenstates and eigenvalues of a fictitious, nontapered grating with the corresponding gap width [i.e., $w \rightarrow w-2 z \tan \theta$ in Eq. (4) with proper boundary conditions]. ${ }^{17}$ We note a formal equivalence with the multislice (or beam propagation) method. Our present approach is exemplified in Fig. 2, in which the amplitude and phase-shift profiles of the wave field at the exit of a phase grating are shown for different tapering angles. The amplitude is particularly sensitive to such subtle differences in $\theta$. This finding should prove useful, e.g., for the application of x-ray diffraction to structural studies on nanopatterned polymers. ${ }^{18}$

In order to verify the results obtained using the coupledwave theory, we have also determined the wave field by solving Eq. (1) directly using a finite-difference method ${ }^{19}$ (shown in Fig. 2 for comparison). A similar theoretical approach has previously been adopted for determining the wave field inside $\mathrm{x}$-ray waveguides. ${ }^{20}$ The remarkable agreement between the two different theoretical approaches should be noted.

Finally, we apply the coupled-wave theory outlined above to phase-retrieval-based diffractive $\mathrm{x}$-ray imaging of a colloidal suspension confined in a microfluidic array. Details of the experiment, carried out at beamline X04SA of the Swiss Light Source, can be found elsewhere. ${ }^{4}$ Within our present approach, the real-space (i.e., support) constraints are applied, in each step of the iterative phase-retrieval algorithm, ${ }^{7}$ to the exit wave field backward propagated through the sample utilizing the eigenmodes of the grating filled with a homogeneous colloidal suspension. This approach effectively removes both the container and the volume-diffraction effects. Hence, the reconstruction yields the ordering-induced phase-shift modulation of the confined colloidal suspension (with respect to the homogeneous counterpart), a quantity directly proportional to the average density modulations within the confining gap. We note that the above theory is formally exact only in the absence of confinement-induced ordering of the colloids. However, since the ordering constitutes a weak phase object compared to the homogeneously filled grating, the present approximation is expected to be valid.

The result of this approach is presented in Fig. 3 as the phase-shift profile across the confining gap for a representative confined colloidal suspension exhibiting ordering in two layers parallel to the interfaces. The slight taper at the interfaces can be attributed to the finite resolution of the experi- 


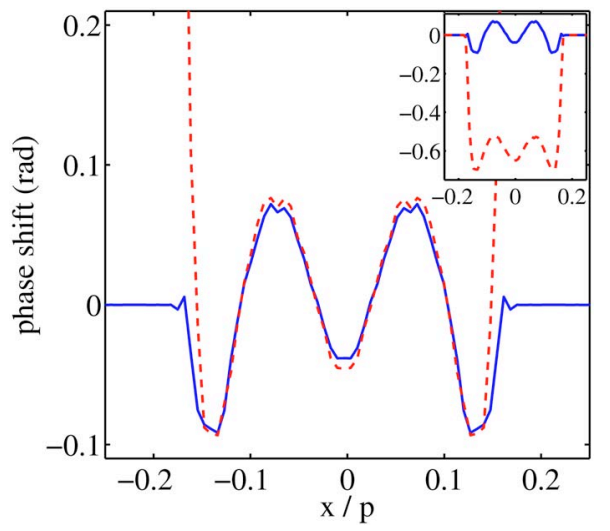

FIG. 3. (Color online) Phase-shift profile across the confining gap for a colloidal suspension as determined from the experimental $\mathrm{x}$-ray diffraction data of Ref. 4. The solid line is obtained using the present approach. The dashed line denotes the corresponding profile (including the container) as given by the approximate approach in Ref. 7 (vertically offset to facilitate comparison). Inset: Unshifted phase profiles.

mental data. Minor remnant volume-diffraction effects can also be observed in the profile, which could be due to the adopted structural parameters of the microfluidic array. ${ }^{21} \mathrm{We}$ further note that our present approach neglects, e.g., interface roughness ${ }^{22}$ and the native oxide layer of the array. The corresponding profile (including the container) as obtained using the ad hoc approach outlined in Ref. 7 is also shown in Fig. 3 for comparison. The excellent agreement between the results of the different approaches in terms of ordering should be noted. However, the present approach describes more accurately the depletion layer close to the interface.

The above inclusion of volume-diffraction effects is obtained at the expense of simplicity. Contrariwise to the model-independent approach of Ref. 7, accurate a priori knowledge about the structural parameters of the microfluidic array is essential. Nevertheless, the approach outlined in the present study should prove useful for future studies on, e.g., micelles or protein solutions in confinement. More generally, an approach similar to the present one for subtracting volume-diffraction effects in diffractive x-ray imaging could be applied to any sample partitionable into a strong known and a weak unknown phase object.
This work was performed at the Swiss Light Source, Paul Scherrer Institut, Villigen, Switzerland. We thank P. Thibault for comments on the manuscript.

${ }^{1}$ J. Miao, P. Charalambous, J. Kirz, and D. Sayre, Nature (London) 400, 342 (1999).

${ }^{2}$ M. A. Pfeifer, G. J. Williams, I. A. Vartanyants, R. Harder, and I. K. Robinson, Nature (London) 442, 63 (2006).

${ }^{3}$ D. Shapiro, P. Thibault, T. Beetz, V. Elser, M. Howells, C. Jacobsen, J. Kirz, E. Lima, H. Miao, A. M. Neiman, and D. Sayre, Proc. Natl. Acad. Sci. U.S.A. 102, 15343 (2005).

${ }^{4}$ O. Bunk, A. Diaz, F. Pfeiffer, C. David, C. Padeste, H. Keymeulen, P. R. Willmott, B. D. Patterson, B. Schmitt, D. K. Satapathy, J. F. van der Veen, H. Guo, and G. H. Wegdam, Phys. Rev. E 75, 021501 (2007).

${ }^{5}$ P. Thibault, V. Elser, C. Jacobsen, D. Shapiro, and D. Sayre, Acta Crystallogr., Sect. A: Found. Crystallogr. 62, 248 (2006).

${ }^{6}$ R. Harder, M. A. Pfeifer, G. J. Williams, I. A. Vartaniants, and I. K. Robinson, Phys. Rev. B 77, 115425 (2007).

${ }^{7}$ O. Bunk, A. Diaz, F. Pfeiffer, C. David, B. Schmitt, D. K. Satapathy, and J. F. van der Veen, Acta Crystallogr., Sect. A: Found. Crystallogr. 63, 306 (2007).

${ }^{8}$ G. Schneider, Appl. Phys. Lett. 71, 2242 (1997).

${ }^{9}$ F. Pfeiffer, C. David, J. F. van der Veen, and C. Bergemann, Phys. Rev. B 73, 245331 (2006).

${ }^{10}$ C. G. Schroer, Phys. Rev. B 74, 033405 (2006).

${ }^{11}$ M. J. Zwanenburg, J. F. Peters, J. H. H. Bongaerts, S. A. de Vries, D. L. Abernathy, and J. F. van der Veen, Phys. Rev. Lett. 82, 1696 (1999).

${ }^{12} \beta / \delta \sim 10^{-2}$ for $\mathrm{Si}$ at the present wavelength.

${ }^{13}$ The eigenstates and eigenvalues for $|m| \leq 70$, are obtained by transforming the coefficient matrix in Eq. (4) to Hessenberg form and solving the linear matrix equation using the QR algorithm (Ref. 23).

${ }^{14}$ C. Fuhse and T. Salditt, Opt. Commun. 265, 140 (2006).

${ }^{15}$ A. Diaz, C. David, H. Guo, H. Keymeulen, F. Pfeiffer, G. Wegdam, T. Weitkamp, and J. F. van der Veen, Physica B 357, 199 (2005).

${ }^{16} \mathrm{D}$. Marcuse, Theory of Dielectric Optical Waveguides, 2nd ed. (Academic, San Diego, 1991).

${ }^{17}$ We use the longitudinal step size $\Delta z=10 \mathrm{~nm}$.

${ }^{18}$ R. L. Jones, T. Hu, E. K. Lin, W.-L. Wu, R. Kolb, D. M. Casa, P. J. Bolton, and G. B. Barclay, Appl. Phys. Lett. 83, 4059 (2003).

${ }^{19}$ We use the Crank-Nicolson scheme (Ref. 23) with step sizes $\Delta x=\Delta z$ $=1 \mathrm{~nm}$.

${ }^{20}$ A. Jarre, C. Fuhse, C. Ollinger, J. Seeger, R. Tucoulou, and T. Salditt, Phys. Rev. Lett. 94, 074801 (2005).

${ }^{21}$ The duty cycle $w / p$, and the tapering angle $\theta$, are determined from the diffraction efficiencies of the empty grating. The other parameters are obtained using the model-independent approach of Ref. 7.

${ }^{22}$ G. Schneider, Appl. Phys. Lett. 73, 599 (1998).

${ }^{23}$ W. H. Press, S. A. Teukolsky, W. T. Vetterling, and B. P. Flannery, Numerical Recipes in FORTRAN: The Art of Scientific Computing, 2nd ed. (Cambridge University Press, Cambridge, 1992). 